\title{
DHA supplementation for late onset Stargardt disease: NAT-3 study
}

This article was published in the following Dove Press journal:

Clinical Ophthalmology

24 June 2010

Number of times this article has been viewed

\section{Giuseppe Querques' \\ Pascale Benlian' \\ Bernard Chanu ${ }^{2}$ \\ Nicolas Leveziel' \\ Gabriel Coscas' \\ Gisele Soubrane' \\ Eric H Souied'}

'Department of Ophthalmology, University of Paris XII, Centre

Hospitalier Intercommunal de Creteil, ${ }^{2}$ Department of Nutrition, University of Paris XII, Hopital Henry Mondor, Creteil, France
Correspondence: Giuseppe Querques Department of Ophthalmology, University of Paris XII, Centre Hospitalier Intercommunal de Creteil, 40 Avenue de Verdun, 94000 Creteil, France

Tel +330 I 45 I7 5222

Fax +330145175266

Email giuseppe.querques@hotmail.it
Background: We analyzed the effects of a docosahexaenoic acid (DHA) supplementation in patients affected with late onset Stargardt disease (STGD).

Methods: DHA (840 mg/day) was given to 20 STGD patients for six months. A complete ophthalmologic examination, including best-corrected visual acuity (BCVA) and multifocal electroretinogram (mfERG), was performed at inclusion day 0 (D0) and at month 6 (M6).

Results: Overall, no statistical differences have been observed at M6 vs D0 as regards BCVA and mfERG $(P>0.05)$. Mild Improvement of BCVA and improvement of mfERG was noted in seven/40 eyes of four/20 patients. In the first patient, the peak of the $a$ wave increased from $66 \mathrm{nV} / \mathrm{deg}^{2}$ to $75.4 \mathrm{nV} / \mathrm{deg}^{2}$ in the right eye (RE) and $24.5 \mathrm{nV} / \mathrm{deg}^{2}$ to $49.1 \mathrm{nV} / \mathrm{deg}^{2}$ in the left eye (LE). The peak of the $b$ wave improved from $122 \mathrm{nV} / \mathrm{deg}^{2}$ to $157 \mathrm{nV} / \mathrm{deg}^{2}$ in the RE, and $102 \mathrm{nV} / \mathrm{deg}^{2}$ to $149 \mathrm{nV} / \mathrm{deg}^{2}$ in the LE. In the second patient peaks of the $a$ and $b$ waves respectively increased from $11.8 \mathrm{nV} / \mathrm{deg}^{2}$ to $72.1 \mathrm{nV} / \mathrm{deg}^{2}$ and $53 \mathrm{nV} / \mathrm{deg}^{2}$ to $185 \mathrm{nV} / \mathrm{deg}^{2}$ in the RE. In the third patient the peak of the $a$ wave increased from $37 \mathrm{nV} / \mathrm{deg}^{2}$ to $43 \mathrm{nV} / \mathrm{deg}^{2}$ in the RE, and from $31 \mathrm{nV} / \mathrm{deg}^{2}$ to $45 \mathrm{nV} / \mathrm{deg}^{2}$ in the LE; the peak of the $b$ wave improved from $70 \mathrm{nV} / \mathrm{deg}^{2}$ to $89 \mathrm{nV} / \mathrm{deg}^{2}$ in the RE, and from $101 \mathrm{nV} / \mathrm{deg}^{2}$ to $108 \mathrm{nV} / \mathrm{deg}^{2}$ in the LE. In the fourth patient, the peak of the $a$ wave increased from $39 \mathrm{nV} / \mathrm{deg}^{2}$ to $42 \mathrm{nV} / \mathrm{deg}^{2}$ in the RE, and from $40 \mathrm{nV} / \mathrm{deg}^{2}$ to $43 \mathrm{nV} / \mathrm{deg}^{2}$ in the LE; the peak of the $b$ wave improved from $86 \mathrm{nV} / \mathrm{deg}^{2}$ to $94 \mathrm{nV} / \mathrm{deg}^{2}$ in the RE, and from $87 \mathrm{nV} / \mathrm{deg}^{2}$ to $107 \mathrm{nV} / \mathrm{deg}^{2}$ in the LE.

Conclusion: DHA seems to influence some functional parameters in patients affected with STGD. However, no short-term benefit should be expected from DHA supplementation.

Keywords: docosahexaenoic acid (DHA), multifocal electroretinogram, omega-3, polyunsaturated fatty acid, retinal dystrophy, Stargardt disease

\section{Background}

Stargardt disease (STGD), described by Karl Stargardt in 1909, ${ }^{1-3}$ and fundus flavimaculatus (FFM), a STGD-like phenotype described by Franceschetti in $1965^{4}$ are variants of the same hereditary disease that affects the retinal pigment epithelium (RPE) and photoreceptor layer, both linked with the $A B C A 4$ gene..$^{5-7}$ When characterized by a juvenile onset (first two decades), a rapidly progressive course, and a poor visual outcome, the disease is usually termed STGD. The term FFM is favored when the disease begins at the end of the second decade or within the third decade, and has a slowly progressive course.$^{8-9}$ The general course of STGD and FFM is a progressive central atrophy, resulting in a central vision loss $(\leq 20 / 200)$. However, to date, the rapidity of visual loss remains unpredictable. ${ }^{10-11}$

The retina has a high concentration of omega-3, and particularly docosahexaenoic acid (DHA). The high concentration of DHA in the photoreceptors cells ( $>50 \%$ of the 
lipids of the external membrane) suggests its major role in the maintenance of the structure of these cells. Moreover, since photoreceptor outer segments require a constant supply of these omega-3 fatty acids owing to their continuous renewal, diets rich in DHA may improve retinal function and slow-down the progressive photoreceptor degeneration. Many studies demonstrated that DHA has a protective role in the retina, ${ }^{12-17}$ increased mitochondrial activity, increased RPE acid lipase activity, antioxidative, antiproliferative, and antiapoptotic effects. ${ }^{18}$

For these reasons, in this study, we decided to analyze the effects of DHA supplementation in patients affected with late onset STGD, an inherited macular dystrophy characterized by progressive photoreceptor degeneration. The current study (NAT-3 study) is part of a clinical trial on nutritional prevention treatment, that we have been performing at our Department to study the preventive effects of DHA on progression of agerelated macular degeneration (Nutritional AMD Treatment, NAT-1 and NAT-2 studies) and other macular diseases.

\section{Methods}

This study was designed as a prospective interventional pilot study. Patients diagnosed with late onset STGD (reported onset at $>18$ years old) were prospectively included. Informed consent was obtained according to a Paris XII University Institutional Review Board - approved protocol. Criteria for inclusion were: 1) age $>18$ years old; 2) evidence of hypo-autofluorescence from areas of macular atrophy, associated or not with retinal flecks; 3) presence of hyperautofluorescent retinal flecks, associated or not with areas of macular atrophy, 4) diagnosis of dark choroid on fluorescein angiography (FA). This study was performed in agreement with the Declaration of Helsinki and French legislation, and was approved by our local ethics committee. In this trial DHA (840 $\mathrm{mg} /$ day) was given to all patients for six months. A complete ophthalmologic examination including best-corrected visual acuity (BCVA), measured at $4 \mathrm{~m}$ with standard Early Treatment Diabetic Retinopathy Study (ETDRS) charts, fundus examination, autofluorescent frames (AF), FA, optical coherence tomography (OCT 3; Humphrey Zeiss, San Leandro, CA), and multifocal electroretinogram (mfERG), was performed at inclusion day 0 (D0) and at month 6 (M6). Molecular biology analysis of ELOVL4 gene was also performed in order to establish predicting factors of efficacy of DHA treatment. In addition, a complete profile of fatty acids in serum (S) and in red blood cell membranes (RBCM) performed by gas chromatography was recorded at D0 and M6.

Statistical calculations were performed using Epinfo 3.3 software package (CDC, Atlanta, GA). The Mann-Whitney/
Wilcoxon two-sample test was used to compare main ophthalmologic findings, and fatty acids in S and in RBCM, at D0 and M6. The chosen level of statistical significance was $P<0.05$.

\section{Results}

Twenty unrelated patients (nine women, eleven men) with clinically definite late onset STGD were included (Table 1). Mean age of patients was $45 \pm 15$ years old (range 24-72). Neither side effects nor dropouts were observed in all patients. Overall, for the included patients, no statistical differences have been observed at M6 vs D0 as regards BCVA and mfERG $(P>0.05)$. Four out of the 20 patients showed a mild improvement of visual acuity (three eyes) and mfERG recordings (seven eyes). In the first patient (case three, a 66 years old man), BCVA improved from 20/25 to 20/20 in the right eye (RE) and from 20/32 to 20/20 in the left eye (LE); the peak of the $a$ wave increased from $66 \mathrm{nV} / \mathrm{deg}^{2}$ to $75.4 \mathrm{nV} / \mathrm{deg}^{2}$ in the RE, and from $24.5 \mathrm{nV} / \mathrm{deg}^{2}$ to $49.1 \mathrm{nV} / \mathrm{deg}^{2}$ in the LE (Figure 1). The peak of the $b$ wave improved from $122 \mathrm{nV} / \mathrm{deg}^{2}$ to $157 \mathrm{nV} / \mathrm{deg}^{2}$ in the RE, and from $102 \mathrm{nV} / \mathrm{deg}^{2}$ to $149 \mathrm{nV} / \mathrm{deg}^{2}$ in the LE (Figure 1). In the second patient (case five, a 72 years old woman), BCVA improved from $20 / 25$ to $20 / 20$ in the RE; the peak of the $a$ wave increased

Table I Patients demographics and BCVA changes from baseline to the six month FU

\begin{tabular}{lllllll}
\hline Case & Gender & Age & \multicolumn{2}{l}{$\begin{array}{l}\text { BCVA at } \\
\text { baseline }\end{array}$} & \multicolumn{2}{l}{$\begin{array}{l}\text { BCVA at } \\
\text { 6-month FU }\end{array}$} \\
\cline { 4 - 7 } & & & RE & LE & RE & LE \\
\hline case I & M & 57 & $20 / 50$ & $20 / 40$ & $20 / 50$ & $20 / 40$ \\
case 2 & F & 30 & $20 / 125$ & $20 / 40$ & $20 / 125$ & $20 / 40$ \\
case 3 & M & 66 & $20 / 25$ & $20 / 32$ & $20 / 20$ & $20 / 20$ \\
case 4 & M & 5 I & $20 / 40$ & $20 / 160$ & $20 / 40$ & $20 / 160$ \\
case 5 & F & 72 & $20 / 25$ & $20 / 40$ & $20 / 20$ & $20 / 40$ \\
case 6 & M & 55 & $20 / 32$ & $20 / 50$ & $20 / 32$ & $20 / 50$ \\
case 7 & F & 24 & $20 / 20$ & $20 / 20$ & $20 / 20$ & $20 / 20$ \\
case 8 & F & 49 & $20 / 100$ & $20 / 80$ & $20 / 100$ & $20 / 80$ \\
case 9 & M & 4 I & $20 / 40$ & $20 / 50$ & $20 / 40$ & $20 / 50$ \\
case 10 & M & 39 & $20 / 25$ & $20 / 32$ & $20 / 25$ & $20 / 32$ \\
case II & M & 25 & $20 / 32$ & $20 / 32$ & $20 / 32$ & $20 / 32$ \\
case 12 & F & 62 & $20 / 64$ & $20 / 50$ & $20 / 64$ & $20 / 50$ \\
case 13 & M & 36 & $20 / 100$ & $20 / 200$ & $20 / 100$ & $20 / 200$ \\
case 14 & M & 30 & $20 / 50$ & $20 / 160$ & $20 / 50$ & $20 / 160$ \\
case 15 & M & 34 & $20 / 64$ & $20 / 25$ & $20 / 64$ & $20 / 25$ \\
case 16 & F & 46 & $20 / 20$ & $20 / 25$ & $20 / 20$ & $20 / 25$ \\
case 17 & F & 62 & $20 / 64$ & $20 / 64$ & $20 / 64$ & $20 / 64$ \\
case 18 & F & 48 & $20 / 32$ & $20 / 50$ & $20 / 32$ & $20 / 50$ \\
case 19 & F & 51 & $20 / 25$ & $20 / 25$ & $20 / 25$ & $20 / 25$ \\
case 20 & M & 26 & $20 / 32$ & $20 / 40$ & $20 / 32$ & $20 / 40$ \\
\hline
\end{tabular}

Abbreviations: BCVA, best-corrected visual acuity; FU, follow-up; M, male; $F$, female; RE, right eye; LE, right eye. 

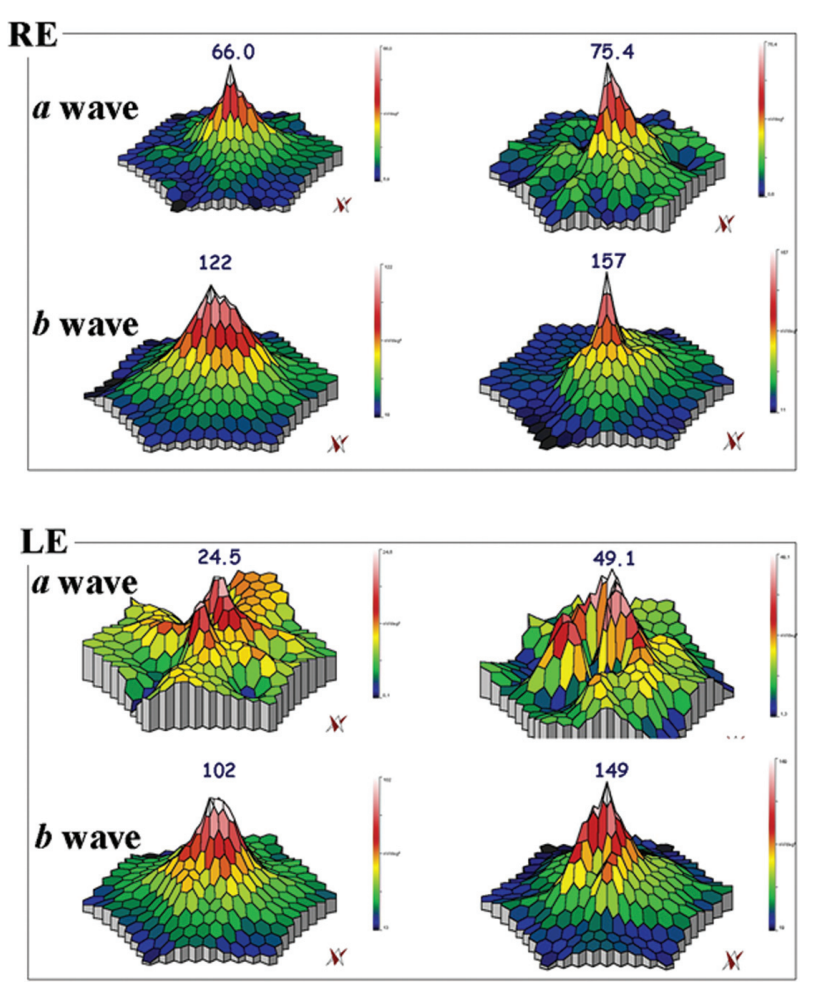

Figure I mfERG showing the peaks of $a$ and $b$ waves for case three. In the RE, the peak of the $a$ wave increased from $66 \mathrm{nV} / \mathrm{deg}^{2}$ (top left panel) to $75.4 \mathrm{nV} / \mathrm{deg}^{2}$ (top right panel), and, in the LE, from $24.5 \mathrm{nV} / \mathrm{deg}^{2}$ (bottom left panel) to $49.1 \mathrm{nV} / \mathrm{deg}^{2}$ (bottom right panel), after six months. The peak of the $b$ wave improved from $122 \mathrm{nV} / \mathrm{deg}^{2}$ (top left panel) to $157 \mathrm{nV} / \mathrm{deg}^{2}$ (top right panel) RE, and from $102 \mathrm{nV} / \mathrm{deg}^{2}$ (bottom left panel) to $149 \mathrm{nV} / \mathrm{deg}^{2}$ (bottom right panel) LE, after six months. Abbreviations: mfERG, multifocal electroretinogram; RE, right eye; LE, left eye.

from $11.8 \mathrm{nV} / \mathrm{deg}^{2}$ to $72.1 \mathrm{nV} / \mathrm{deg}^{2}$ in the $\mathrm{RE}$, and the peak of the $b$ wave improved from $53 \mathrm{nV} / \mathrm{deg}^{2}$ to $185 \mathrm{nV} / \mathrm{deg}^{2}$ in the RE (Figure 2). The LE was affected with choroidal neovascularization (CNV), and the patient was treated twice by photodynamic therapy. No improvement was observed on BCVA and the foveal function. Nevertheless, it is notable that the four external rings slightly improved (Figure 2). In the third patient (case seven, a 24 years old woman), BCVA was 20/20 in both eyes at baseline, and remained unchanged throughout the six month period; the peak of the $a$ wave increased from $37 \mathrm{nV} / \mathrm{deg}^{2}$ to $43 \mathrm{nV} / \mathrm{deg}^{2}$ in the RE, and from $31 \mathrm{nV} / \mathrm{deg}^{2}$ to $45 \mathrm{nV} / \mathrm{deg}^{2}$ in the LE (Figure 3 ). The peak of the $b$ wave improved from $70 \mathrm{nV} / \mathrm{deg}^{2}$ to $89 \mathrm{nV} / \mathrm{deg}^{2}$ in the RE, and from $101 \mathrm{nV} / \mathrm{deg}^{2}$ to $108 \mathrm{nV} / \mathrm{deg}^{2}$ in the LE (Figure 3). In the fourth patient (case 11, a 25 years old man), BCVA was 20/32 in both eyes at baseline, and remained unchanged throughout the six-month period; the peak of the $a$ wave increased from $39 \mathrm{nV} / \mathrm{deg}^{2}$ to $42 \mathrm{nV} / \mathrm{deg}^{2}$ in the RE, and from $40 \mathrm{nV} / \mathrm{deg}^{2}$ to $43 \mathrm{nV} / \mathrm{deg}^{2}$ in the LE (Figure 4 ). The peak of the $b$ wave improved from $86 \mathrm{nV} / \mathrm{deg}^{2}$ to $94 \mathrm{nV} / \mathrm{deg}^{2}$ in the RE, and from $87 \mathrm{nV} / \mathrm{deg}^{2}$ to $107 \mathrm{nV} / \mathrm{deg}^{2}$ in the LE (Figure 4).
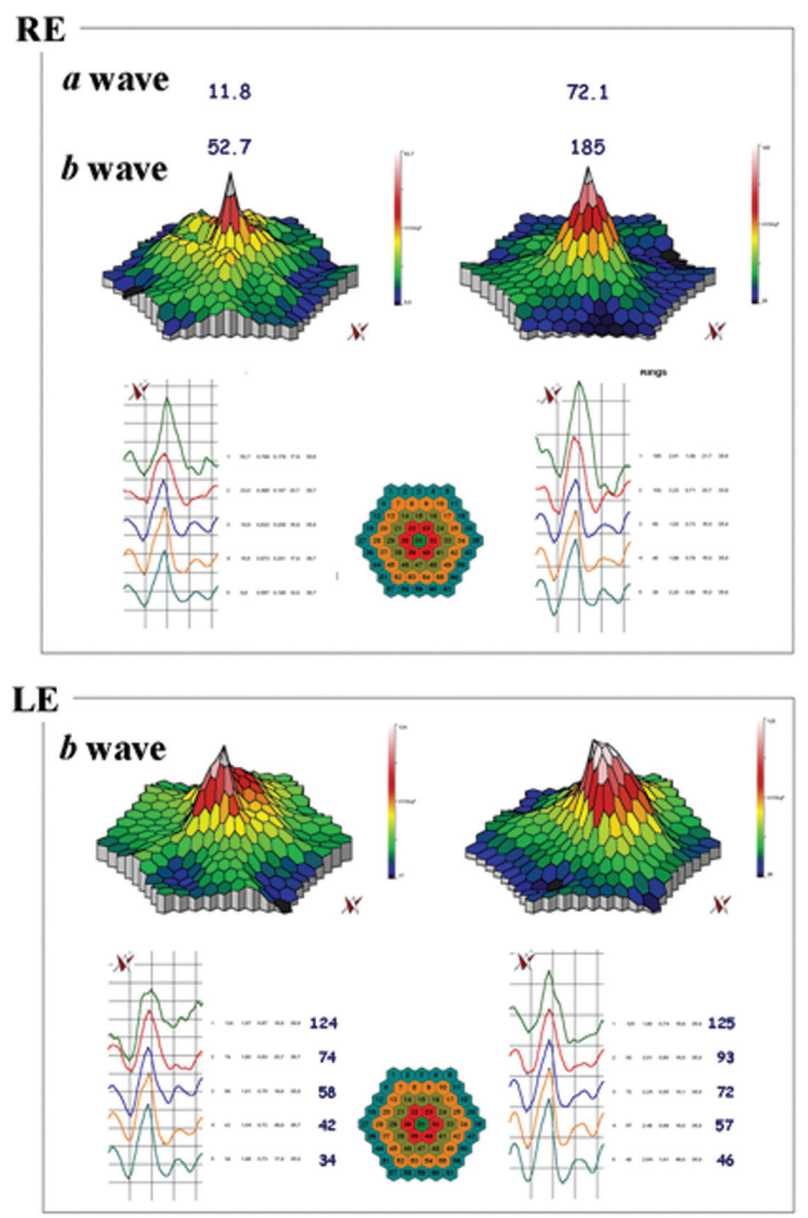

Figure 2 mfERG showing the peaks of $a$ and $b$ waves for case five. In the RE the peaks of the $a$ and $b$ waves respectively increased from $11.8 \mathrm{nV} / \mathrm{deg}^{2}$ (top right panel) to $72.1 \mathrm{nV} / \mathrm{deg}^{2}$ (top left panel) and from $53 \mathrm{nV} / \mathrm{deg}^{2}$ (top right panel) to $185 \mathrm{nV} / \mathrm{deg}^{2}$ (top left panel), after six months. The LE was affected with choroidal neovascularization: it is notable that the four external ring were improved when comparing before (bottom right panel) and after treatment (bottom left panel). Abbreviations: mfERG, multifocal electroretinogram; RE, right eye; LE, left eye.

During this short period of time, no progression was observed in the size of the central atrophy. Moreover, FA revealed no progression to CNV in any of the 20 patients within the six month period. Molecular biology analysis of ELOVL4 gene which was performed to establish predicting factors of efficacy of DHA treatment, revealed the absence of ELOVL4 gene mutations in all patients. Five patients were explored for their plasma lipids before and after DHA supplementation. These patients showed significant changes, with a $106 \%$ increase in S DHA $(1.5 \%$ at D0 vs up to $2.7 \%$ at M6, $P=0.013$ ), and: a $37 \%$ increase in RBCM DHA (4.0\% at D0 up to 5.2 at M6, $P=0.035)$. Other fatty acids were unchanged in $\mathrm{S}$ or RBCM.

There was no evidence of systemic illness or toxic effects attributable to DHA during the course of this study. 


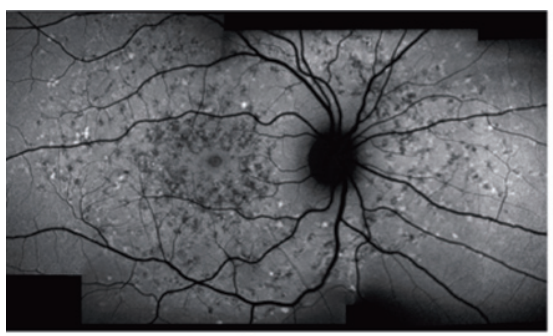

a wave $37 \mathrm{nV} / \mathrm{deg}^{2}$
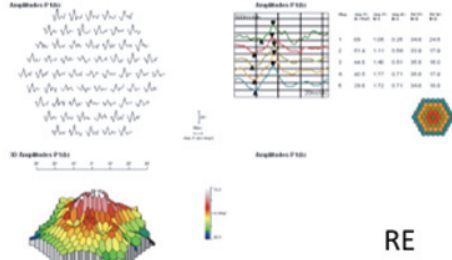

a wave $43 \mathrm{nV} / \mathrm{deg}^{2}$
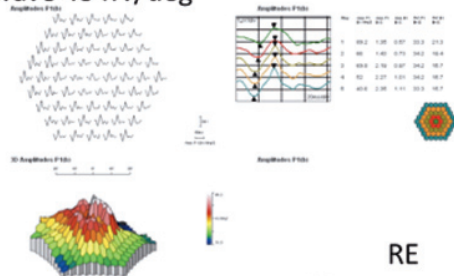

RE

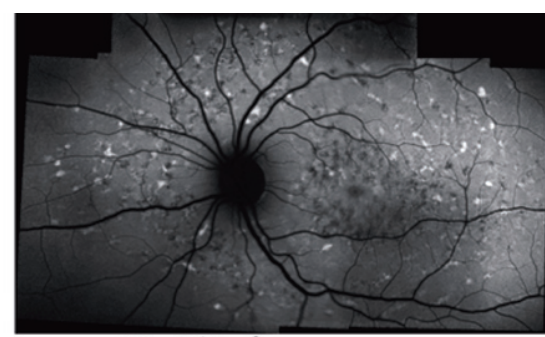

a wave $31 \mathrm{nV} / \mathrm{deg}^{2}$

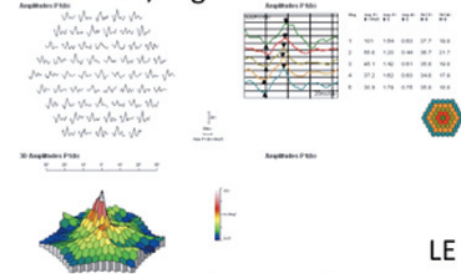

a wave $45 \mathrm{nV} / \mathrm{deg}^{2}$

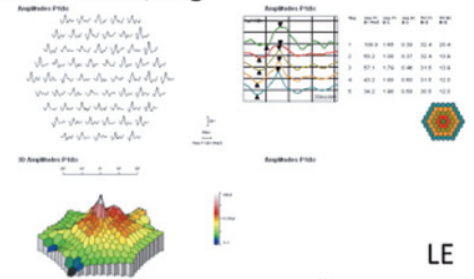

LE

Figure 3 Fundus autofluorescent frames (upper left and right panels) and mfERG for case seven. The peak of the $a$ wave increased from $37 \mathrm{nV} / \mathrm{deg}^{2}$ to $43 \mathrm{nV} / \mathrm{deg}^{2}$ in the RE, and from $3 \mathrm{I} \mathrm{nV/deg}{ }^{2}$ to $45 \mathrm{nV} / \mathrm{deg}^{2}$ in the LE. The peak of the $b$ wave improved from $70 \mathrm{nV} / \mathrm{deg}^{2}$ (middle left panel) to $89 \mathrm{nV} / \mathrm{deg}^{2}$ (bottom left panel) in the RE, and from

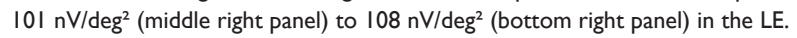

Abbreviations: mfERG, multifocal electroretinogram; RE, right eye; LE, left eye.

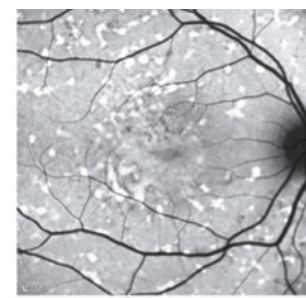

a wave $39 \mathrm{nV} / \mathrm{deg}^{2}$

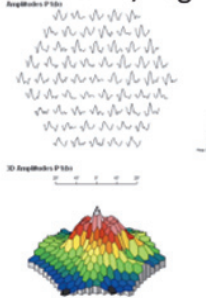

a wave $42 \mathrm{nV} / \mathrm{deg}^{2}$
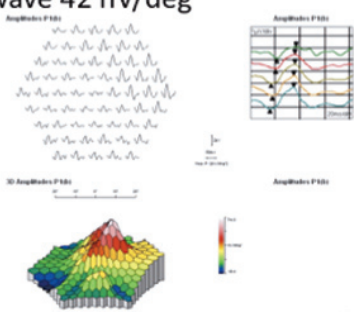

$\mathrm{RE}$

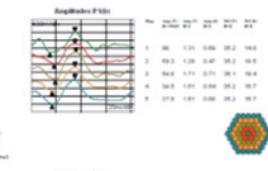

\section{西}




\section{Discussion}

MacDonald et $\mathrm{al}^{19}$ recently demonstrated the efficacy of DHA in a 15 years old girl affected with autosomal dominant STGD macular dystrophy associated with the ELOVL4 gene mutation. ELOVL4 is homologous to a fatty acid elongase presumably involved in the biosynthesis of DHA. An animal study previously demonstrated that the dietary level of n-3 fatty acids not only affects the level of DHA, but also the levels of very-long-chain fatty acids in rod outer segments (ROS) membranes..$^{20}$ This young patient was given a dietary supplementation of DHA ( $20 \mathrm{mg} / \mathrm{kg}$ body weight/day) on two occasions, and a clear improvement was observed on visual function both subjectively (visual acuity, VF-14) and objectively (mfERG amplitudes). No conclusion can be drawn from one single case but this case report provides some hope for patients affected with STGD macular dystrophy associated with ELOVL4 mutations and a larger and longer study is warranted.

Hubbard et $\mathrm{al}^{21}$ investigated the influence of dietary factors on the phenotypic severity in a family with autosomal dominant STGD associated with ELOVL4 mutation. Red blood cell membrane and adipose tissue lipids were analyzed as an indication of short-term and long-term dietary fatty acid intake. The authors found a significant inverse relationship between phenotypic severity and levels of eicosapentaenoic acid and docosahexaenoic acid. These results indicate that the phenotypic diversity may be related to differences in dietary fat intake as reflected by adipose and red blood cell lipids.

In our series, molecular biology analysis of ELOVL4 gene, which was performed in order to establish predicting factors of efficacy of DHA treatment, revealed the absence of ELOVL4 gene mutations in all the 20 patients. Despite this absence of ELOVL4 gene mutations (excluding that the direct disease-causing mechanism might be the ELOVL4 gene involvement in fatty acid elongation in the retina), four out of 20 patients had an improvement of visual acuity and improvement of mfERG.

DHA is known to have specific retinal effects such as increased mitochondrial activity, increased RPE acid lipase activity, antioxidative, antiproliferative, and antiapoptotic effects. ${ }^{18}$ DHA has also shown to inhibit eicosanoid synthesis from arachidonic acid and cytokine production form macrophages. ${ }^{22}$ This antiinflammatory effect could play a beneficial role in the retina as the pathway of inflammation is suggested to be involved in the occurrence of several retinal diseases. Moreover, since photoreceptor outer segments require a constant supply of these omega- 3 fatty acids due to their continuous renewal (DHA represents the main component of the lipids of the external membrane of the photoreceptors cells, suggesting a major role in the maintenance of the structure of these cells), diets rich in DHA may improve retinal function and slow-down the progressive photoreceptor degeneration.

Of note, in our series, patients who had regular DHA intake during six months, and that were explored for their plasma lipids before and after DHA supplementation, displayed a strong and significant increase in serum and erythrocyte membrane DHA. Therefore, it may be that, DHA supplementation, mainly due to antiinflammatory, antioxidative and antiapoptotic properties, ${ }^{18}$ could have a protective effect in some cases of STGD whatever the genotype.

Mild improvement of BCVA and/or improvement of mfERG were noted in four/20 cases. Moreover, during this short period of time, no progression was observed either in the size of the central atrophy, or to CNV, in any of the 20 patients. There was no evidence of systemic illness or a toxic effect that was attributable to DHA during the course of this study.

Our study is flawed by several shortcomings: the sample size was small, with no control group, and relatively short follow-up. No major improvement was observed in this series, given that only a mild effect was recorded, even on the four improved cases, we could not rule out normal inter-examination variations of mfERG. Therefore, larger series and analysis of both ABCA4 and ELOVL4 genes is needed.

\section{Conclusion}

DHA seems to influence some functional parameters in patients affected with STGD however, no short-term benefit should be expected from DHA supplementation.

\section{Acknowledgments}

Giuseppe Querques and Eric H Souied were involved in the conception and design of the study; Giuseppe Querques, Pascale Benlian, Bernard Chanu, Nicolas Leveziel and Eric H Souied were involved in the analysis of the data; Giuseppe Querques, Gabriel Coscas, Gisele Soubrane and Eric $\mathrm{H}$ Souied, were involved in the preparation of the manuscript. The authors report no conflicts of interest in this work.

\section{References}

1.Stargardt K. Ueber familiare progressive degeneration in der makulagegend des Auges. Albrecht V Graefes. Arch Ophthalmol. 1909;71:534-550. 
2. Cibis GW, Morey M, Karris DJ. Dominantly inherited macular dystrophy with flecks (Stargardt). Arch Ophthalmol. 1980;98(10):1785.

3. Merlin S, Landau J. Abnormal findings in relatives of patients with juvenile hereditary macular degeneration (Stargardt's disease). Ophthalmologica. 1970;161(1):1.

4. Franceschetti A, Francois J. Fundus flavimaculatus. Arch Ophthalmol. 1965;25:505-530.

5. Allikmets R, Singh N, Sun H, et al. A photoreceptor cell-specific ATPbinding transporter gene (ABCR) is mutated in recessive Stargardt macular dystrophy. Nat Genet. 1997;15(3):236-246.

6. Cremers FP, van de Pol DJ, van Driel M, et al. Autosomal recessive retinitis pigmentosa and cone-rod dystrophy caused by splice site mutations in the Stargardt's disease gene ABCR. Hum Mol Genet. 1998;7(3):355-362.

7. Rozet JM, Gerber S, Souied E, et al. Spectrum of ABCR gene mutations in autosomal recessive macular dystrophies. Eur J Hum Genet. 1998;6(3):291-295.

8. Aaberg TM. Stargardt's disease and fundus flavimaculatus: evaluation of morphologic progression and intrafamilial co-existence. Trans Am Ophthalmol Soc. 1980;84:453-487.

9. Lois N, Holder GE, Bunce C, Fitzke FW, Bird AC. Phenotypic subtypes of Stargardt macular dystrophy-fundus flavimaculatus. Arch Ophthalmol. 2001;119(3):359-369.

10. Ergun E, Hermann B, Wirtitsch M. Assessment of central visual function in Stargardts' disease/fundus flavimaculatus with ultrahigh-resolution optical coherence tomography. Invest Ophthalmol Vis Sci. 2005;46(1):310-316.

11. Hargitai J, Zernant J, Somfai GM, et al. Correlation of clinical and genetic findings in Hungarian patients with Stargardt disease. Invest Ophthalmol Vis Sci. 2005;46:4402-4408.
12. Kagan VE, Shvedova AA, Novikov KN. Participation of phospholipases in the "repair" of photoreceptor membranes subjected to peroxidation. Biofizika. 1978;23(2):279-84.

13. Terman A, Brunk UT. Lipofuscin: mechanisms of formation and increase with age. APMIS. 1998;106(2):265-76.

14. Wolman M. Oxidation of lipids and membranes I: in vitro formation of peroxidative lipid polymers. J Supramol Struct. 1975;3:80-9.

15. Tappel AL. Lipid peroxidation damage to cell components. Fed Proc. 1973;32(8):1870-4.

16. Feeney L, Berman ER. Oxygen toxicity: membrane damage by free radicals. Invest Ophthalmol. 1976;15(10):789-92.

17. Keys SA, Boley E, Zimmerman WF. A model membrane system to investigate antioxidants in bovine rod outer segments. Exp Eye Res. 1997;64(3):313-21.

18. Rotstein NP, Politi LE, German OL, Girotti R. Protective effect of docosahexaenoic acid on oxidative stress-induced apoptosis of retina photoreceptors. Invest Ophthalmol Vis Sci. 2003;44(5):2252-2259.

19. MacDonald IM, Hebert M, Yau RJ, et al. Effect of docosahexaenoic acid supplementation on retinal function in a patient with autosomal dominant Stargardt-like retinal dystrophy. Br J Ophthalmol. 2004;88(2):305-306.

20. Xi ZP, Wang JY. Effect of dietary n-3 fatty acids on the composition of long- and very-long-chain polyenoic fatty acid in rat retina. J Nutr Sci Vitaminol. 2003;49(3):210-213.

21. Hubbard AF, Askew EW, Singh N, Leppert M, Bernstein PS. Association of adipose and red blood cell lipids with severity of dominant Stargardt macular dystrophy (STGD3) secondary to an ELOVL4 mutation. Arch Ophthalmol. 2006;124(2):257-263.

22. James MJ, Gibson RA, Cleland LG. Dietary polyunsaturated fatty acids and inflammatory mediator production. Am J Clin Nutr. 2000;71(1 Suppl):343S-348S.
Clinical Ophthalmology

\section{Publish your work in this journal}

Clinical Ophthalmology is an international, peer-reviewed journal covering all subspecialties within ophthalmology. Key topics include: Optometry; Visual science; Pharmacology and drug therapy in eye diseases; Basic Sciences; Primary and Secondary eye care; Patient Safety and Quality of Care Improvements. This journal is indexed on

\section{Dovepress}

PubMed Central and CAS, and is the official journal of The Society of Clinical Ophthalmology (SCO). The manuscript management system is completely online and includes a very quick and fair peer-review system, which is all easy to use. Visit http://www.dovepress.com/ testimonials.php to read real quotes from published authors. 